\title{
Efficacy, Safety, and Pharmacokinetics of a Novel Human Immune Globulin Subcutaneous, 20 \% in Patients with Primary Immunodeficiency Diseases in North America
}

\author{
Daniel Suez $^{1} \cdot$ Mark Stein $^{2} \cdot$ Sudhir Gupta $^{3} \cdot$ Iftikhar Hussain $^{4} \cdot$ Isaac Melamed $^{5}$. \\ Kenneth Paris ${ }^{6}$ - Amy Darter ${ }^{7}$. Christelle Bourgeois ${ }^{8} \cdot$ Sandor Fritsch $^{8} \cdot$ Heinz Leibl $^{8}$. \\ Barbara $\mathrm{McCoy}^{9} \cdot$ David Gelmont $^{10} \cdot{\text { Leman } \mathrm{Yel}^{3,9}}^{3}$
}

Received: 30 May 2016/Accepted: 5 August 2016/Published online: 31 August 2016

(C) The Author(s) 2016. This article is published with open access at Springerlink.com

\begin{abstract}
Patients with primary immunodeficiency disease (PIDD) typically require life-long intravenous (IV) or subcutaneous (SC) immunoglobulin (Ig) replacement therapy to prevent recurrent infections. The efficacy, safety, and pharmacokinetics of a highly concentrated (20\%) Ig preparation for SC administration (IGSC $20 \%$ ) were evaluated in a prospective trial in patients with PIDD. A total of 74 patients (aged 383 years) received 4327 IGSC $20 \%$ infusions over a median of 380.5 days. The rate of validated serious bacterial infections was 0.012 event/patient-year $(p<0.0001$ compared with the historical control), and the annualized rate of infection was
\end{abstract}

Electronic supplementary material The online version of this article (doi:10.1007/s10875-016-0327-9) contains supplementary material, which is available to authorized users.

Leman Yel

Leman.Yel@shire.com

Allergy, Asthma and Immunology Clinic PA, Irving, TX, USA

2 Allergy Associates of the Palm Beaches, North Palm Beach, FL, USA

3 Division of Basic and Clinical Immunology, University of California at Irvine, Irvine, CA, USA

4 Vital Prospects Clinical Research Institute, Tulsa, OK, USA

5 IMMUNOe International Research Centers, Centennial, CO, USA

6 LSU Health Sciences Center, Children's Hospital, New Orleans, LA, USA

7 Oklahoma Institute of Allergy and Asthma Clinical Research, LLC, Oklahoma City, OK, USA

8 Baxalta Innovations $\mathrm{GmbH}$, now part of Shire, Vienna, Austria

9 Baxalta US Inc., now part of Shire, 650 East Kendall Street, Cambridge, MA, USA

10 Baxalta US Inc., now part of Shire, Westlake Village, CA, USA
2.41 events/patient. Median IgG trough levels were $>14.5 \mathrm{~g} /$ 1 . The median maximum infusion rate was $60 \mathrm{ml} / \mathrm{h} / \mathrm{site}$ (range 4.4-180), resulting in a median infusion duration of $0.95 \mathrm{~h}$. A volume $\geq 30 \mathrm{ml}$ was infused per site in $74.8 \%$ of IGSC $20 \%$ infusions. Most (84.9\%) infusions were administered using $\leq 2$ infusion sites; for $99.8 \%$ of infusions, there was no need to interrupt/stop administration or reduce the infusion rate. No related serious adverse event (AE) occurred during IGSC $20 \%$ treatment; related non-serious AEs occurred at a rate of 0.036 event/infusion. The incidence of related local AEs was 0.015 event/infusion and of related systemic AEs was 0.021 event/infusion; most were mild in severity, none severe. Increased infusion rates or volumes were not associated with higher AE rates. The investigated IGSC $20 \%$ treatment was shown to be effective and safe, enabling higher infusion rates and volumes per site compared to conventional SC treatments, resulting in fewer infusion sites and shorter infusion durations.

Keywords Primary immunodeficiency diseases . Immunoglobulin replacement therapy · Subcutaneous administration $\cdot 20 \%$ immunoglobulin $\cdot$ Pharmacokinetics

\section{Introduction}

Primary immunodeficiency diseases (PIDD) result from genetic defects in the immune system, more than 300 of which have been identified [1]. Patients with PIDD are susceptible to bacterial, viral, and fungal infections [2]. In about $53 \%$ of these patients, the diagnosis of PIDD is associated with defective antibody production with or without decreased levels of serum immunoglobulin (Ig) (e.g., common variable immunodeficiency, specific antibody deficiency, X-linked or 
autosomal recessive agammaglobulinemia, hyper IgM syndrome) [3].

Polyclonal IgG preparations purified from human plasma have been used as antibody replacement therapy to reduce the number and severity of infections in patients with PIDD since the early 1950 s $[4,5]$. Due to their diverse specificity, polyclonal IgGs are able to neutralize infectious agents, enhance phagocytosis, and modulate the immune response. For clinical effectiveness in PIDD, antibody replacement therapy generally requires a monthly dose in the range of 0.3 to $0.6 \mathrm{~g} / \mathrm{kg}$ body weight (BW) administered intravenously (IV) or subcutaneously (SC) [6]. With IgG solutions at 5 or $10 \%$ weight per volume $(w / v)$ protein concentration, volumes of 3 to $6 \mathrm{ml} / \mathrm{kg}$ BW are routinely administered IV [7].

IgG replacement therapy administered SC (IGSC) has been widely established in recent years. It is safe and well-tolerated with a particularly low risk of systemic adverse reactions compared to IV administration of IgG [5, 7]. IGSC slowly diffuses from the SC space into the systemic circulation while equilibrating with the extravascular compartment. Consequently, there is no high peak in the IgG concentration as seen following IV infusion, and sustained steady-state IgG levels can be achieved. At the same monthly equivalent dose as IV immunoglobulin replacement therapy (IGIV), IGSC may lead to higher serum IgG trough levels compared to IV infusion [8, 9]. With appropriate training by healthcare professionals, SC infusions of immunoglobulin can easily be performed by patients at home without assistance, thus increasing their comfort and independence and also reducing cost [10].

A drawback of IGSC compared to IV infusion is the limited volume that can be administered into each site due to resistance of the SC extracellular matrix. Consequently, the rate at which IGSC can be infused is slower compared to IGIV, resulting in longer infusion times and requiring multiple infusion sites per treatment, usually on a weekly basis. One of the strategies to overcome these limitations is the development of highly concentrated IgG formulations that allow for infusion of the same dose in smaller infusion volumes compared to less concentrated products [5].

Alternatively, SC immunoglobulin product that could be infused at higher rates and volumes per site would provide an advantage over currently available conventional SC preparations by decreasing infusion duration and the number of infusion sites. Immune globulin subcutaneous (human) (IGSC $20 \%$, Baxalta, now part of Shire) is a ready-for-use, sterile liquid preparation of highly purified, concentrated, functionally intact human IgG developed specifically for SC administration to provide patients with an additional treatment option.

Presented here are the results of a prospective phase $2 / 3$ study that evaluated the efficacy, safety, tolerability, and pharmacokinetic (PK) characteristics of a new IGSC $20 \%$ treatment option in adult and pediatric patients with PIDD in North America.

\section{Methods}

\section{Study Design}

This prospective, open-label clinical trial (registered on clinicaltrials.gov: NCT no. 01218438) was conducted in accordance with the Declaration of Helsinki and the international standards of Good Clinical Practice. Patients were enrolled at 15 sites in the USA and Canada; informed consent was obtained from each patient prior to undergoing any study procedures.

The trial comprised four study periods: in period 1 , patients received IGIV $10 \%$ and in periods 2 to 4 , they received IGSC $20 \%$ (Supplementary material Figure S1). Period 1 was designed mainly to determine the area under the $\mathrm{IgG}$ concentration curve (AUC) following IV administration $\left(\mathrm{AUC}_{\mathrm{IV}}\right)$. During IGSC $20 \%$ treatment, systemic exposure equivalent to previous IGIV $10 \%$ treatment (as measured by the AUC of total IgG over time) was targeted. Therefore, IGSC $20 \%$ doses were adjusted to compensate for the lower bioavailability of IgG-administered SC. The adjustment factor to calculate the IGSC $20 \%$ dose in period $2(145 \%)$ was approximated from the PK data of available IGSC products $[11,12]$. Next, based on the PK data collected from the first 18 patients treated with IGSC $20 \%$ at $145 \%$ of the IGIV $10 \%$ dose in period 2 , the IGSC $20 \%$ dose that would (on average) provide equivalent IgG exposure as IGIV $10 \%$ administration ("adjusted IGSC $20 \%$ dose") was determined to be $145 \%$ of the IGIV $10 \%$ dose. In period 3, patients were treated with IGSC $20 \%$ at the "adjusted dose." Since this adjusted dose represented the average dose-response of only 18 patients, the possibility of over- or under-dosing could not be excluded. Thus, for each patient, an individually adapted (individualized) dose of IGSC $20 \%$ was determined by comparing the patient trough level (equivalent to the steady-state serum IgG level) attained in period 3 to the expected trough level increase calculated from the PK data of periods 1 and 2. During period 4, the patients were infused with IGSC $20 \%$ at this dose for 40 weeks.

\section{Study Population}

Patients aged 2 years and older diagnosed with PIDD involving defective antibody production and requiring IgG replacement as defined by the International Union of Immunological Societies (IUIS) Scientific Committee 2011 [13] and by diagnostic criteria according to Conley et al. [14] were eligible for enrollment in the study. Inclusion criteria also required that patients had received a stable dose (IV or SC) of IgG equivalent to at least $0.3 \mathrm{~g} / \mathrm{kg} \mathrm{BW} / 4$ weeks and not higher than $1 \mathrm{~g} / \mathrm{kg}$ $\mathrm{BW} / 4$ weeks for a minimum of 12 weeks prior to first treatment and had serum IgG trough levels $>5 \mathrm{~g} / \mathrm{l}$ at screening. Patients were excluded from the study if they had a history of hepatitis $\mathrm{B}$ or $\mathrm{C}$ or a positive human immunodeficiency 
virus test; if they had persistent abnormal alanine aminotransferase and aspartate aminotransferase values $>2.5$ times the upper limit of normal for the testing laboratory, creatinine clearance value $<60 \%$ of normal according to their age and gender, or severe neutropenia or protein loss at screening; or if they had been diagnosed with a malignancy, were receiving anticoagulation therapy, or had a history of thrombotic episodes. Patients were also excluded if they were receiving antibiotics, had an active infection at the time of screening, or had had an acute serious bacterial infection within 3 months prior to screening. A complete list of eligibility criteria is available in the Supplementary material.

\section{Study Product}

IGSC $20 \%$ is a liquid concentrate of functionally intact, aggregate-free IgG derived from human plasma. The production of IGSC $20 \%$ follows the same manufacturing processes as IGI, $10 \%$ solution (marketed under the Baxalta, now part of Shire trade-name GAMMAGARD LIQUID ${ }^{\circledR}$ in the US and Kiovig ${ }^{\circledR}$ in the EU) except for ultra/diafiltration and final formulation at $20 \%(w / v)$ protein concentration. The manufacturing process of IGSC $20 \%$ includes three dedicated virus inactivation and reduction steps: solvent/detergent (S/D) treatment [15], nanofiltration $(35 \mathrm{~nm})[15,16]$, and low $\mathrm{pH}$ incubation with elevated temperature [17, 18]. Similar to IGI, $10 \%$, IGSC $20 \%$ contains glycine as stabilizer to minimize IgG dimerization. The final IGSC $20 \%$ product has a viscosity of $14.4 \mathrm{mPa} / \mathrm{s}$, an osmolality of $280-292 \mathrm{mOsm} / \mathrm{kg}$ and contains trace amounts of IgA (average concentration $80 \mu \mathrm{g} /$ $\mathrm{ml}$ ). Each lot of IGSC $20 \%$ is monitored for procoagulant activity using a thrombin generation assay to ensure that the final container is free of procoagulants.

\section{Immunoglobulin Treatments}

Patients received IGIV $10 \%$ at the monthly equivalent dose used prior to entering the study (required dose range 0.3$1.0 \mathrm{~g} / \mathrm{kg} \mathrm{BW} / 4$ weeks) every 3 or 4 weeks at the clinical site. IGSC $20 \%$ was administered once a week; doses used in the respective study periods are described in the "Study design" section. IGSC $20 \%$ was infused using an electromechanical syringe-driver pump (CME T34L, Caesarea Medical Electronics) and high-flow, 24-gauge low-resistance needles (RMS Medical Products). The needle sets used in the trial ranged from 6 to $12 \mathrm{~mm}$ in length at the discretion of the investigator; there was no specified needle length for infusion. Infusion rates were increased incrementally: the initial two infusions were to be started at $10 \mathrm{ml} / \mathrm{h} /$ infusion site and could be increased to a maximum of $20 \mathrm{ml} / \mathrm{h} / \mathrm{infu}-$ sion site. Subsequent infusions could begin at the maximum tolerated infusion rate for the initial infusions, and as tolerated, the infusion rate was to be increased to a maximum of
$60 \mathrm{ml} / \mathrm{h} / \mathrm{site}$. For patients with a body weight of $40 \mathrm{~kg}$ or above, an infusion volume of up to $60 \mathrm{ml}$ was to be administered per infusion site if well tolerated. For patients with a body weight below $40 \mathrm{~kg}$, it was recommended to limit infusion volume to $20 \mathrm{ml}$ per site for the initial two infusions. Volumes could then be increased to a maximum of $60 \mathrm{ml}$ per site as tolerated. Multiple infusion sites could be used simultaneously. Infusion sites were to be rotated to avoid any single infusion site being used repeatedly within a short time interval. Infusion of IGSC $20 \%$ at home was possible after sufficient training of the patient/caregiver or with assistance of a healthcare professional.

\section{Efficacy Assessment}

Efficacy was evaluated based on the analysis of serious acute bacterial infections, all infections, and $\operatorname{IgG}$ levels. Serious bacterial infections, such as bacteremia/sepsis, bacterial meningitis, osteomyelitis/septic arthritis, bacterial pneumonia, and visceral abscesses caused by a recognized bacterial pathogen were diagnosed according to the Diagnostic Criteria for Serious Infection Types in the FDA Guidance for Industry, June 2008 [19]. The primary efficacy assessment was the annualized rate of validated acute serious bacterial infections (VASBIs, defined as $\mathrm{R}_{\mathrm{VASBI}}=$ mean number of VASBI/patient/year). Efficacy was also evaluated by the annualized rate of all infections (i.e., VASBIs and all other events clinically assessed as infections), as well as the number of fever episodes (body temperature $\geq 38^{\circ} \mathrm{C}$ ), the number of days with fever, the number of days missed from school/work or to perform normal daily activities due to illness/infection, the number of admissions to a hospital as an in-patient, and the number of days as a stationary patient in the hospital, as well as urgent or unscheduled physician visits due to illness/infection (apart from the regular investigator/study site visits scheduled every $8-12$ weeks within the study).

\section{Safety}

Safety was evaluated through clinical and laboratory assessments. Safety data were collected throughout the study. The AEs that occurred during the infusions at the site (every 8 12 weeks) were recorded by the investigator. All investigators were specifically trained on symptoms of potential AEs. All patients received an eDiary tablet to continuously record home treatments, AEs, and additional information as they occurred. The investigator provided guidance for the patient/caregiver regarding identification and documentation of local and systemic AEs, including signs of hemolysis such as fever, chills, back pain, fatigue, and dark urine. All patients were instructed to inform the investigator/site immediately in case of such an event. In addition, the patient was contacted by the investigator within 3-5 days after each infusion, either at the study site 
or at their home for follow-up to ensure appropriate documentation of AEs. The investigators reviewed patients' eDiary entries at every site visit. All AEs were assessed by the investigator using comprehensive data collection systems - including the patient's eDiary-for seriousness, severity, temporal association, and possible causal relatedness to the investigational product.

Monitoring for potential cases of hemolysis comprised routine hematology screening and hemolysis screening as recommended by the FDA Guidance for Industry (June 2008 [19]). If a decrease of hemoglobin $\geq 2 \mathrm{~g} / \mathrm{dl}$ was measured during either the hematology or hemolysis screening, the assessments to monitor for potential cases of hemolysis were to be performed within 48-72 $\mathrm{h}$ of being informed of the hemoglobin level, unless there was a clear alternative explanation. These assessments included direct antiglobin (Coomb's) test, plasma-free hemoglobin, reticulocyte count, lactate dehydrogenase (LDH), serum haptoglobin, and urine hemosiderin.

\section{Pharmacokinetics}

Determination of total serum IgG concentration was performed at a central laboratory using a validated enzymelinked immunosorbent assay (ELISA)-based method. PK assessments were performed over a specific dosing interval: in period 1 between infusions 10 and 11, for patients aged 12 years and older; in period 2 between infusions 9 and 10, for the first 18 patients aged 12 years and older; and for all patients in period 4 between infusions 17 and 18 (Supplementary material Figure S1). In period 2 and 4, samples were collected preinfusion and on days 1, 3, 5, and 7 after IGSC $20 \%$ administration in patients aged 12 years and older. In patients aged 2-11 years, samples to determine the AUC over a dosing interval ( $\mathrm{AUC}_{0-\tau}$ ) after IGSC $20 \%$ administration were only drawn preinfusion and on days 3 and 7 in order to limit the number of blood draws.

Total serum IgG trough levels were assessed immediately prior to each IGIV $10 \%$ infusion in period 1 and during IGSC $20 \%$ treatment: prior to infusion 1, twice each in periods 2 and 3 , four times during period 4 , and at the end-of-study visit.

\section{Statistical Methods}

Efficacy was assessed by the mean number of VASBIs per patient per year $\left(\mathrm{R}_{\mathrm{VASBI}}\right)$. Assuming $\mathrm{R}_{\mathrm{VASBI}}=0.6$ and a onesided test and a Type I error $=0.01$, a sample size of 59 patients would have in excess of $85 \%$ power to test the null hypothesis that $\mathrm{R}_{\mathrm{VASBI}} \geq 1.0$ against the alternative hypothesis $\mathrm{R}_{\text {VASBI }}<1.0 . \mathrm{R}_{\text {VASBI }}$ and $99 \%$ upper confidence limit (CI) were calculated using a Poisson model accounting for the length of the observation periods per patient.

The AUC between adjacent infusions was calculated by the trapezoidal rule. To allow for comparisons between periods 1 ,
2 , and $4, \mathrm{AUC}_{0-} \tau$ was standardized for the infusion intervals ( 3 or 4 weeks vs. 1 week $=\mathrm{AUC}_{0-\tau ; \text {; }}$;). The bioavailability of IGSC $20 \%$ relative to IGIV $10 \%$ was estimated from the ratio of $\mathrm{AUC}_{0-\tau ; \mathrm{h}}$ in period 4 ( $\mathrm{SC}$ treatment at an individualized dose, once per week) over $\mathrm{AUC}_{0-\tau ; \mathrm{h}}$ in period 1 (IV treatment every 3 or 4 weeks) standardized to 1 week.

\section{Measures of Patient Experience}

Treatment burden related to Ig therapy was evaluated with the Life Quality Index questionnaire (LQI) for the age group 2 to 12 years (observer: parent) and the age group 13 years and older (observer: patient) [20, 21]. The LQI covers four domains: treatment interference, therapy-related problems, therapy settings, and treatment costs. While data related to treatment cost LQI domain were collected, they were not included in the analysis as patients received free treatment during the study. Treatment satisfaction was surveyed in age groups from 2 to 12 years (observer: parent) and 13 years and older (observer: patient) using the Treatment Satisfaction Questionnaire for Medication (TSQM-9) [22]. Evaluations were performed at baseline, at the end of periods 1 and 3 and during the end-of-study visit (or early termination visit). Score changes between end of period 1 and end of period 3 or end of study visit were analyzed. In both the LQI and TSQM9 , higher scores indicated higher satisfaction.

\section{Results}

\section{Study Population}

Seventy-seven (77) patients with PIDD started study period 1 (51.9\% males, $48.1 \%$ females; age range 3-83 years; Supplementary material Table S1). More than half of the patients had either common variable immunodeficiency $(33.7 \%)$, "specific antibody deficiency" (23.4\%), or agammaglobulinemia (14.3\%, congenital and autosomal recessive combined; Supplementary material Table S2). All patients had received antibody replacement therapy until just prior to study entry (68.8\% IV; $31.2 \% \mathrm{SC})$. None of the patients screened for inclusion in the study had elevated transaminases, and therefore, no patient was excluded based on these criteria. Overall, 74 patients were administered IGSC $20 \%$ and 67 (90.5\%) patients completed the study (Supplementary material Figure S2). Two patients were discontinued from the study during period 1 for non-adherence, and one patient withdrew from the study after being hospitalized for mild headache (SAE) assessed as related to IGIV $10 \%$ infusion. During IGSC $20 \%$ treatment, seven patients prematurely discontinued treatment. One patient who had common variable immune deficiency and fibromyalgia experienced fatigue (unrelated to IGSC $20 \%$ administration) and chose to discontinue. One patient was terminated from the study 
because of poor adherence to the study protocol and five patients withdrew consent for reasons unrelated to AEs (Supplementary material Figure S2).

\section{Efficacy}

During IGSC $20 \%$ treatment, the point estimate of $\mathrm{R}_{\mathrm{VASBI}}$ was statistically significantly lower than $1\left(\mathrm{R}_{\mathrm{VASBI}}=0.01\right.$; $99 \% \mathrm{CI}=0.024 ; p<0.0001$; Table 1$)$. One VASBI of pneumonia treated with systemic antibiotics developed during period 4 in a 78-year-old patient who had specific antibody deficiency and a history of allergic bronchopulmonary aspergillosis.

The point estimate of the annualized rate of all infections was 2.41 events/patient during IGSC $20 \%$ treatment and 3.86 events/patient during IGIV $10 \%$ administration (Table 1). While receiving IGSC $20 \%$, the annualized rate of days off of school/work was 1.16 days, and hospitalizations occurred at a rate of less than once per year for $\leq 1$ day/year (all point estimates). Overall, 58/74 (78.4 \%) patients received antibiotics mostly for treatment of acute infections during IGSC $20 \%$ for an annualized duration of 57.59 days (point estimate). The point estimate of the rate of acute (urgent or unscheduled) physician visits due to infection or other illness was also less than one visit per year (Table 1).

\section{Safety}

IGSC $20 \%$ was safe, with no serious causal-related AEs. Of the three serious AEs that occurred during the trial, one was a mild headache assessed as related to IGIV $10 \%$ by the investigator for which the patient was hospitalized and kept under observation and subsequently withdrew from the study. The other two SAEs, a severe lung adenocarcinoma and a moderate pneumonia, the VASBI described above, were not deemed related to IGSC $20 \%$ treatment.

The incidence of non-serious AEs per infusion was 0.108 event/infusion during IGSC $20 \%$ treatment and was 0.556 event/infusion during IGIV $10 \%$ administration (Table 2). Of the 466 non-serious AEs (other than infections) reported for IGSC $20 \%, 157$ non-serious AEs (0.036 event/infusion) were deemed causally related to IGSC $20 \%$; most (136/157; $86.6 \%$ ) were of mild severity; none were severe.

Systemic AEs assessed as causally related to IGSC $20 \%$ treatment were reported in $25.7 \%$ of patients with an incidence of 0.021 event/infusion. The most frequent systemic AEs considered related to IGSC $20 \%$ infusions were headache $(0.011$ event/infusion) followed by fatigue and nausea (0.002 event/infusion each; Table 3). Headache was experienced by $10.8 \%$ of patients receiving IGSC $20 \%$ infusion. Diarrhea was reported by $2.7 \%$ of patients, however with an incidence of less than 0.001 per infusion. The other systemic AEs deemed related to IGSC $20 \%$ were reported at a very low frequency $(\leq 0.001$ event/ infusion, Table 3$)$. There was no event of laboratory-confirmed hemolysis following IGSC $20 \%$ administration. A decline in hemoglobin of $2.0 \mathrm{~g} / \mathrm{dl}$ or more was observed in six patients (during IGIV $10 \%$ treatment $(n=1)$, during IGSC $20 \%$ administration $(n=3)$, and at the "end-of-study" visit $(n=2))$. However, at no time was there a concordance of other laboratory test results (e.g.,
Table 1 Efficacy of protection against infections

\begin{tabular}{|c|c|c|c|c|}
\hline \multirow[t]{3}{*}{ Parameter } & \multicolumn{4}{|c|}{ Annualized rate $\mathrm{a}^{\mathrm{a}}$ per patient per treatment } \\
\hline & \multicolumn{2}{|c|}{ IGIV $10 \%(19.67 \text { PY })^{\mathrm{b}}$} & \multicolumn{2}{|c|}{ IGSC $20 \%(83.70 \mathrm{PY})^{\mathrm{b}}$} \\
\hline & Point estimate & $95 \% \mathrm{CI}$ & Point estimate & $95 \% \mathrm{CI}$ \\
\hline $\begin{array}{l}\text { Validated acute bacterial infections } \\
\text { (VASBIs) [upper limit } 99 \% \mathrm{CI} \text { ] }\end{array}$ & $0.00[0.234]$ & 0.00 to 0.19 & $0.01^{\mathrm{c}}[0.024]$ & 0.01 to 0.02 \\
\hline All infections ${ }^{d}$ & 3.86 & $2.77-5.22$ & 2.41 & $1.89-3.03$ \\
\hline Sinus infections & 0.97 & $0.61-1.45$ & 0.69 & $0.50-0.93$ \\
\hline Fever episodes & 0.61 & $0.34-0.99$ & 0.13 & $0.08-0.21$ \\
\hline $\begin{array}{l}\text { Days off school or work due to } \\
\text { illness or infection }\end{array}$ & 3.20 & $1.88-5.03$ & 1.16 & $0.70-1.79$ \\
\hline Days on antibiotics & 63.2 & $43.39-88.29$ & 57.59 & $40.71-78.59$ \\
\hline Days in hospital & 0.20 & $0.08-0.42$ & 0.11 & $0.05-0.20$ \\
\hline Hospitalizations & 0.05 & $0.02-0.10$ & 0.02 & $0.01-0.04$ \\
\hline Acute physician /emergency room visits & 1.73 & $1.03-2.68$ & 0.86 & $0.54-1.28$ \\
\hline
\end{tabular}


Table 2 Summary of AE analyses

\begin{tabular}{|c|c|c|c|c|}
\hline \multirow[t]{3}{*}{ AE categories } & \multicolumn{4}{|l|}{ Treatments } \\
\hline & \multicolumn{2}{|l|}{ IGIV $10 \%$} & \multicolumn{2}{|l|}{ IGSC $20 \%$} \\
\hline & $\begin{array}{l}\text { Number }(\%) \text { of } \\
\text { patients }(n=77)\end{array}$ & $\begin{array}{l}\text { Number (rate) })^{\mathrm{a}} \text { of } \\
\text { AEs }(n=324)\end{array}$ & $\begin{array}{l}\text { Number }(\%) \text { of } \\
\text { patients }(n=74)\end{array}$ & $\begin{array}{l}\text { Number }(\text { rate })^{\mathrm{a}} \text { of } \\
\text { AEs }(n=4327)\end{array}$ \\
\hline Non-serious AEs (excluding infections) & $51(66.2)$ & $180(0.556)$ & $57(77.0)$ & $466(0.108)$ \\
\hline Mild & $43(55.8)$ & $141(0.435)$ & $53(71.6)$ & $360(0.083)$ \\
\hline Moderate & $17(22.1)$ & $37(0.114)$ & $31(41.9)$ & $104(0.024)$ \\
\hline Severe & $2(2.6)$ & $2(0.006)$ & $2(2.7)$ & $2(<0.001)$ \\
\hline Causally related non-serious AEs & $28(36.4)$ & $80(0.247)$ & $28(37.8)$ & $157(0.036)$ \\
\hline Mild & $22(28.6)$ & $59(0.182)$ & $24(32.4)$ & $136(0.031)$ \\
\hline Moderate & $9(11.7)$ & $19(0.059)$ & $9(12.2)$ & $21(0.005)$ \\
\hline Severe & $2(2.6)$ & $2(0.006)$ & 0 (NA) & 0 (NA) \\
\hline $\begin{array}{l}\text { Causally related local non-serious } \\
\text { AEs (excluding infections) }\end{array}$ & $2(2.6)$ & $2(0.006)$ & $18(24.3)$ & $67(0.015)$ \\
\hline Mild & $1(1.3)$ & $1(0.003)$ & $16(21.6)$ & $62(0.014)$ \\
\hline Moderate & $1(1.3)$ & $1(0.003)$ & $3(4.1)$ & $5(0.001)$ \\
\hline Severe & 0 (NA) & 0 (NA) & 0 (NA) & 0 (NA) \\
\hline $\begin{array}{l}\text { Causally related systemic non-serious } \\
\text { AEs (excluding infections) }\end{array}$ & $27(35.1)$ & $78(0.241)$ & $19(25.7)$ & $90(0.021)$ \\
\hline Mild & $22(28.6)$ & $58(0.179)$ & $16(21.6)$ & $74(0.017)$ \\
\hline Moderate & $8(10.4)$ & $18(0.056)$ & $8(10.8)$ & $16(0.004)$ \\
\hline Severe & $2(2.6)$ & $2(0.006)$ & 0 (NA) & 0 (NA) \\
\hline SAEs (including infections) & $1(1.3)$ & $1(0.003)$ & $2(2.7)$ & $2(<0.001)$ \\
\hline Mild & $1(1.3)$ & $1(0.003)$ & 0 (NA) & 0 (NA) \\
\hline Moderate & 0 (NA) & 0 (NA) & $1(1.35)$ & $1(<0.001)$ \\
\hline Severe & 0 (NA) & 0 (NA) & $1(1.35)$ & $1(<0.001)$ \\
\hline Causally related SAEs & $1(1.3)$ & $1(0.003)$ & 0 (NA) & 0 (NA) \\
\hline Causally related AEs leading to discontinuation & $1(1.3)$ & $1(0.003)$ & 0 (NA) & 0 (NA) \\
\hline AEs leading to death & 0 (NA) & 0 (NA) & 0 (NA) & 0 (NA) \\
\hline
\end{tabular}

$n$ total number of patients or total number of infusions, $A E$ adverse event, $N A$ not applicable; $S A E$ serious AE

${ }^{a}$ Rate per infusion $=$ total number of AEs divided by the total number of infusions

Coomb's test, haptoglobin, free hemoglobin, LDH, urine hemosiderin) supporting a diagnosis of hemolysis in these patients. No renal AEs or changes in laboratory values that measure kidney function were reported during the study.

Causally related local AEs occurred at a frequency of 0.015 event/infusion during IGSC $20 \%$ treatment (Table 2); only $58 / 4327$ (1.3\%) IGSC $20 \%$ infusions were associated with one or more related local AEs. Over the entire course of the study, $24.3 \%$ of patients reported experiencing one or more local AEs related to IGSC $20 \%$ infusion at some time during the study (Table 2). However, the proportion of patients reporting one or more related local $\mathrm{AE}$ and the incidence of related local AEs per patient per year decreased throughout the study. At infusion 1, $13 \%$ of the patients reported one or more related local AE; by infusion 6 , this proportion dropped to below $5 \%$ of the patients and continued to decrease to around $2 \%$ or below and remained at this level until the end of the study (Supplementary material Figure S3A). In addition, the annualized rate of related local AEs was highest in the first 4 weeks of IGSC $20 \%$ treatment and decreased progressively thereafter to values below 1 after 16 weeks of treatment (Supplementary material Figure S3B).

\section{IGSC $20 \%$ Administration Characteristics}

IGSC $20 \%$ was administered to 74 patients for a median treatment duration of 380.5 days (range 30-629). At least one IGSC $20 \%$ infusion was performed at home in $95.9 \%$ of the patients, with or without professional assistance. Patients received a total of 4327 infusions of IGSC $20 \%$ during the study, $79.1 \%$ (3421/4327) of which were administered at home. Of note, certain visits had to be performed at the study site; therefore, even patients who had transitioned to home care were required per protocol to 
Table 3 Causally related nonserious AEs during the IGSC $20 \%$ treatment

\begin{tabular}{lll}
\hline Adverse event $^{\mathrm{a}}$ & \% of patients ${ }^{\mathrm{c}} N=74$ & Rate per infusion $^{\mathrm{d}} N=4327$ \\
\hline Causally related $^{\mathrm{b}}$ systemic AEs & 25.7 & 0.021 \\
Headache & 10.8 & 0.011 \\
Fatigue & 6.8 & 0.002 \\
Nausea & 6.8 & 0.002 \\
Diarrhea & 2.7 & $<0.001$ \\
Myalgia & 4.1 & 0.001 \\
Dizziness & 2.7 & 0.001 \\
Migraine & 2.7 & $<0.001$ \\
Somnolence & 2.7 & $<0.001$ \\
Abdominal pain lower & 1.4 & $<0.001$ \\
Anti-GAD antibody positive & 1.4 & $<0.001$ \\
Pain & 1.4 & $<0.001$ \\
Pruritus & 1.4 & $<0.001$ \\
Causally related ${ }^{\mathrm{b}}$ local AEs & 24.3 & 0.016 \\
Infusion site erythema (including Injection & 10.8 & 0.005 \\
$\quad$ site erythema) & & \\
Infusion site pain (including Infusion site discomfort & 16.2 & 0.008 \\
and Injection site pain) & & $<0.001$ \\
Infusion site pruritus (including Injection site pruritus) & 4.1 & $<0.001$ \\
Infusion site urticaria & 2.7 & $<0.001$ \\
Burning sensation & 1.4 & $<0.001$ \\
Infusion site edema & 1.4 & $<0.001$ \\
Urticaria & 1.4 & \\
\hline
\end{tabular}

${ }^{\mathrm{a}} \mathrm{AEs}$ excluding infections

${ }^{\mathrm{b}}$ Related AE as assessed by the investigator. Missing relationships were treated as related

$\mathbf{c}_{\%} \%$ of patients $=($ total number of affected patients divided by the total number of patients under treatment $) \times 100$

${ }^{\mathrm{d}}$ Rate per infusion $=$ total number of AEs divided by the total number of infusions under treatment receive some infusions on site. The mean $( \pm \mathrm{SD})$ weekly dose of IGSC $20 \%$ was $0.222 \pm 0.071 \mathrm{~g} / \mathrm{kg} /$ week. Across all age groups, a median infusion volume per site of $39.50 \mathrm{ml}$ (range 6.4-76.0) was administered (Table 4). For $74.8 \%$ (3228/4314) of IGSC $20 \%$ infusions, a volume $\geq 30 \mathrm{ml}$ was infused per site. A volume of $60 \mathrm{ml}$ and above per site was administered to $10.8 \%$ of patients at least once (Fig. 1a) and in $7.4 \%(320 / 4314)$ of IGSC $20 \%$ infusions (Fig. 2a). Infusions with a volume per site $<60 \mathrm{ml}$ usually had either a total required dose per infusion of $<60 \mathrm{ml}$ (one site) or a total volume of $>60 \mathrm{ml}$ and $<120 \mathrm{ml}$ and therefore were divided into two sites.

The median maximum rate of infusion was $60 \mathrm{ml} / \mathrm{h} / \mathrm{site}$ (range 4.4-180) resulting in a median infusion duration of $0.95 \mathrm{~h}$ (range 0.2-6.4; Table 4). Overall, $71.6 \%$ of patients achieved a maximum infusion rate of $60 \mathrm{ml} / \mathrm{h} /$ site or more at least once (Fig. 1b) and $57.5 \%$ (2480/4314) of infusions were administered at this infusion rate (Fig. 2b). A median number of 2.0 sites/infusion (range 1-4) were used for administration; $84.9 \%(3662 / 4314)$ of infusions used two infusion sites or fewer.

\section{Tolerability}

The short-term tolerability of IGSC $20 \%$ treatment was evaluated by recording infusions for which the infusion rate had to be reduced, interrupted, or stopped due to tolerability concerns or AEs. For $99.8 \%$ of IGSC $20 \%$ infusions, there was no need to stop/interrupt administration or reduce the infusion rate (Table 5). The infusion rate had to be reduced in five $(0.1 \%)$ IGSC $20 \%$ infusions administered to four patients, two of whom were children (aged 10 and 13 years, respectively). As a result of infusion leakage, two other pediatric patients (aged 8 and 11 years) each had one infusion interrupted, and one infusion was stopped in the 8-year old patient. IGSC $20 \%$ infusions associated with a causally related local AE were categorized by volume and maximum infusion rate per site; the incidence of causally related local AEs did not increase at higher maximum infusion rates and infusion volumes (Fig. 2). Therefore, overall, a very strong positive tolerability profile for IGSC $20 \%$ treatment was demonstrated with infusion rates and volumes of up to $60 \mathrm{ml} / \mathrm{h} / \mathrm{site}$ and $60 \mathrm{ml}$ per site, respectively. 
Table 4 Administration characteristics for IGSC $20 \%$ by age group

\begin{tabular}{|c|c|c|c|c|c|c|}
\hline \multirow[t]{2}{*}{ Parameters $^{\mathrm{a}}$} & \multicolumn{5}{|l|}{ Age group } & \multirow[t]{2}{*}{ All patients $(n=74$} \\
\hline & $\begin{array}{l}2 \text { to }<5 \\
\text { years }(n=1)\end{array}$ & $\begin{array}{l}5 \text { to }<12 \\
\text { years }(n=14)\end{array}$ & $\begin{array}{l}12 \text { to }<16 \\
\text { years }(n=8)\end{array}$ & $\begin{array}{l}16 \text { to }<65 \\
\text { years }(n=45)\end{array}$ & $\begin{array}{l}65 \text { years and } \\
\text { older }(n=9)\end{array}$ & \\
\hline \multicolumn{7}{|c|}{ Duration of infusions (h) } \\
\hline Infusions $(n)$ & 50 & 718 & 346 & 2614 & 434 & 4162 \\
\hline Median & 0.95 & 0.73 & 1.18 & 0.97 & 0.91 & 0.95 \\
\hline Min; $\max$ & $(0.5 ; 1.4)$ & $(0.3 ; 3.45)$ & $(0.3 ; 3.5)$ & $(0.2 ; 4.2)$ & $(0.5 ; 6.4)$ & $(0.2 ; 6.4)$ \\
\hline \multicolumn{7}{|c|}{ Number of sites per infusion } \\
\hline Infusions $(n)$ & 52 & 753 & 360 & 2700 & 461 & 4326 \\
\hline Median & 1 & 2.0 & 2.0 & 2.0 & 2.0 & 2.0 \\
\hline Min; $\max$ & $(1 ; 1)$ & $(1 ; 3)$ & $(1 ; 3)$ & $(1 ; 4)$ & $(1 ; 2)$ & $(1 ; 4)$ \\
\hline \multicolumn{7}{|c|}{ Maximum infusion rate per site $(\mathrm{ml} / \mathrm{h} /$ site $)$} \\
\hline Infusions $(n)$ & 52 & 749 & 360 & 2692 & 461 & 4314 \\
\hline Median & 15.0 & 30.0 & 50.0 & 60.0 & 60.0 & 60.0 \\
\hline Min; $\max$ & $13.5 ; 20.0$ & $4.4 ; 80.0$ & $20.0 ; 120.0$ & $10.0 ; 180.0$ & $5.0 ; 60.0$ & $4.4 ; 180.0$ \\
\hline \multicolumn{7}{|c|}{ Infusion volume per site (ml/site) } \\
\hline Infusions $(n)$ & 52 & 749 & 360 & 2692 & 461 & 4314 \\
\hline Median & 14.5 & 19.5 & 42.7 & 45.3 & 39.0 & 39.5 \\
\hline Min; $\max$ & $13.5 ; 15.5$ & $6.4 ; 43.0$ & $19.2 ; 67.5$ & $18.1 ; 76.0$ & $31.8 ; 56.5$ & $6.4 ; 76.0$ \\
\hline
\end{tabular}

${ }^{\text {a }}$ Only infusions with complete infusion parameters have been considered for each analyses

\section{Pharmacokinetic Parameters}

The pharmacokinetics of serum IgG during IGSC $20 \%$ treatment is depicted in Supplementary material Figure S4. During weekly IGSC $20 \%$ administration at $145 \%$ of the IGIV $10 \%$ dose and at the individualized dose, no IgG peak was observed at day 1 postinfusion, and mean serum IgG levels remained constant throughout the treatment interval (Supplementary material Figure S4). Pharmacokinetic parameters determined for IGSC $20 \%$ and IGIV $10 \%$ are summarized in Table 6 . The bioavailability of IGSC $20 \%$ following 1.45 dose conversion and individual adjustment relative to IGIV $10 \%$ was 1.09 ( $90 \% \mathrm{CI} 1.04$ to $1.13, n=49$ ) as determined from the ratio of the geometric means of the AUC while on IGSC $20 \%$ treatment once per week, compared to IGIV $10 \%$ infusions (standardized to one week).

\section{Total Serum IgG Trough Levels}

Throughout IGSC $20 \%$ treatment, median serum IgG trough values attained at the end of each treatment period remained above $14.5 \mathrm{~g} / \mathrm{L}$ (Table 7). After 17 consecutive weeks of IGSC $20 \%$ treatment at the individualized dose once per week, the median serum IgG trough levels were $15.23 \mathrm{~g} / \mathrm{L}(95 \%$ CI $13.59-15.70 ; n=64)$. The median serum IgG trough levels recorded at the end of IGIV $10 \%$ treatment administered every 3 weeks was $12.0 \mathrm{~g} / 1(95 \%$
CI $11.0-14.1 n=19)$ and was $10.2 \mathrm{~g} / 1$ (95\% CI 9.61$11.3 ; n=50)$ at the end of IGIV $10 \%$ treatment given every 4 weeks (Table 7 ).

\section{Patient Experience}

Changes in treatment satisfaction were assessed for all patients when switching from IGIV $10 \%$ in period 1 to IGSC $20 \%$ in period 3 (adjusted dose) and at the end of period 4 (individualized dose).

Immunoglobulin-related treatment burden was evaluated with the LQI questionnaire in three domains: treatment interference, therapy-related problems, and therapy settings. No significant change in any of the domains was reported in patients aged 2-12 years. For patients 13 years and above, an improvement in each of the domains was observed between period 1 (IGIV $10 \%$ administration) and the subsequent periods on IGSC $20 \%$ treatment. Improvement in the treatment interference domain was found to be statistically significant $(p=0.008)$ across all age groups.

Assessment of treatment satisfaction using the TSQM-9 questionnaire found a significant improvement in the convenience domain $(p<0.001)$ between period 1 (IGIV $10 \%$ administration) and period 4 (IGSC $20 \%$ treatment). No significant improvement in the perception of effectiveness and global satisfaction was observed in either age group: 2-12 years and 13 years and older. 


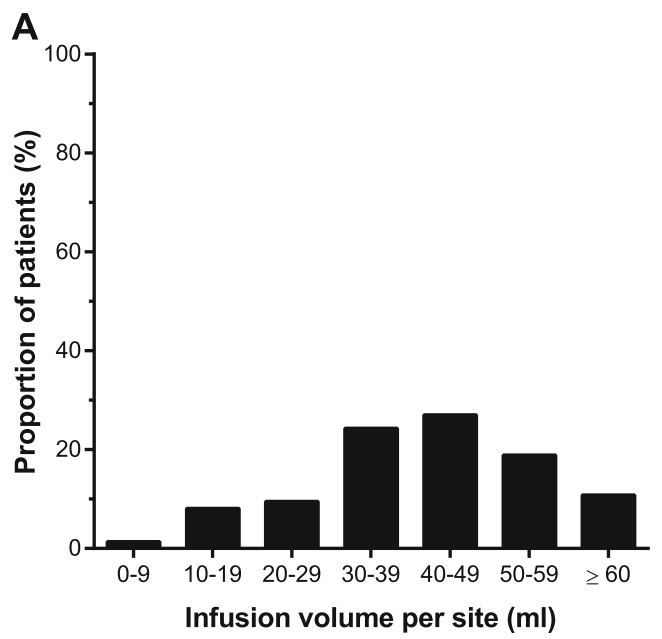

B

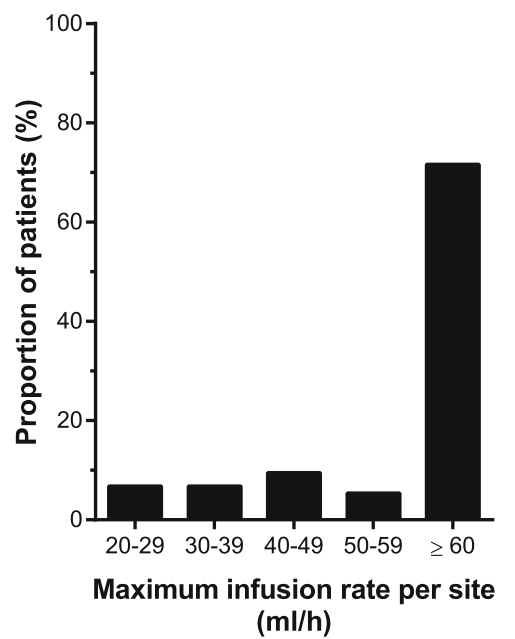

Fig. 1 Categorization of patients by maximum infusion rate and infusion volume. a Infusion volume per site achieved at least once. b Maximum infusion rate achieved at least once

\section{Discussion}

Subcutaneous (SC) administration of human polyclonal immunoglobulin preparations has been shown to be efficacious in preventing infection in patients with PIDD and is associated with fewer systemic adverse reactions compared to the IV route $[5,7,23]$. The improved systemic tolerability and sustained protection from infections observed are likely due to lower peak and higher trough levels of serum IgG administered SC [24]. Drawbacks, however, of SC compared to IV administration with the current standard of 5 and $10 \% \operatorname{IgG}$ preparations are that only small volumes can be infused and multiple infusion sites are required per week for treatment [5, 7]. A highly concentrated $\mathrm{IgG}$ preparation such as the present IGSC $20 \%$ product may offer a new replacement therapy option for patients with PIDD as relatively large volumes per site could be infused at higher rates without impairing tolerability, thereby reducing infusion duration and number of infusion sites.

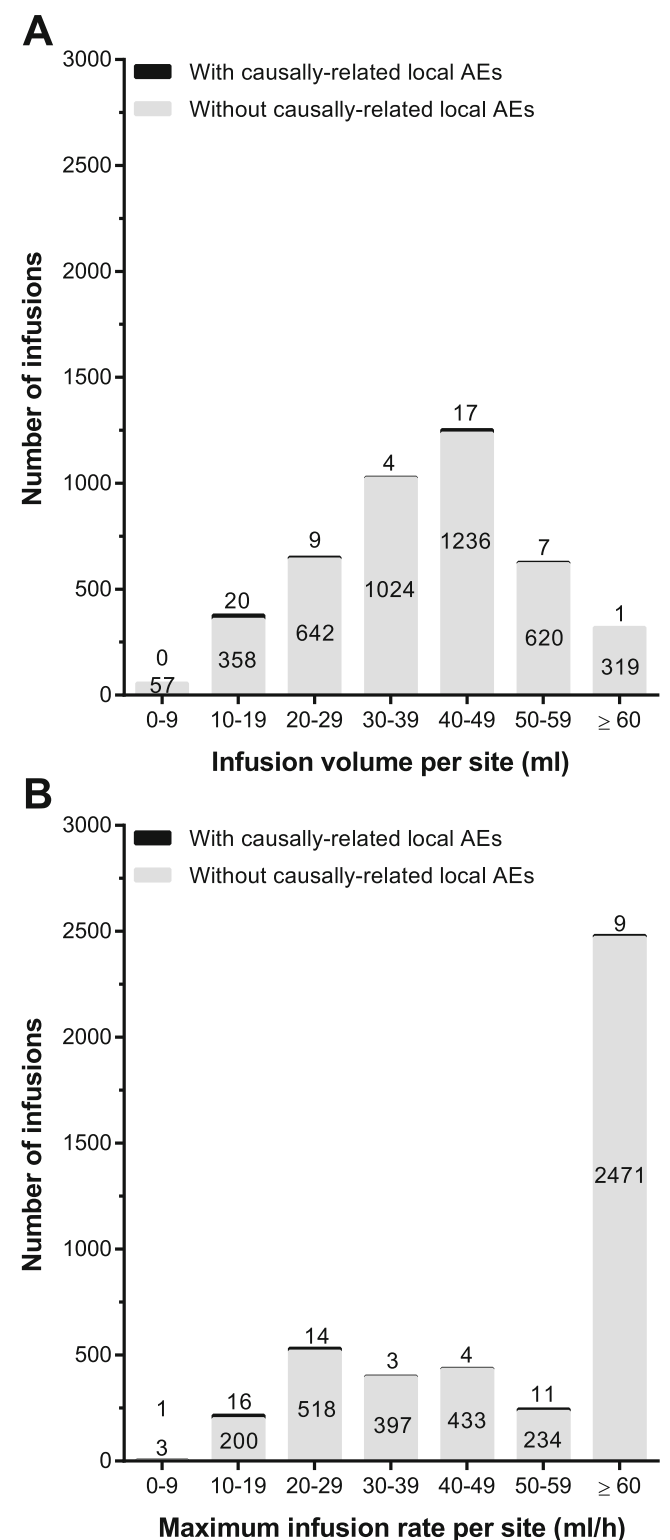

Fig. 2 Tolerability of IGSC $20 \%$ infusion rates and infusion volumes. a Infusion volumes. b Infusion rates. Numbers above the bars indicate the number of infusions associated with a causally related local AE and numbers inside the bars indicate the number of infusions not associated with any causally related local AE. Only infusions with complete infusion history $(n=4314)$ have been considered for these analyses

In this trial, a systemic exposure equivalent to the previous IGIV $10 \%$ treatment was targeted; thus, the IGSC $20 \%$ dose administered was adjusted to compensate for the lower bioavailability of IgG when administered SC [12]. SC administration of individually tailored IGSC $20 \%$ doses was efficacious in providing systemic exposure similar to that obtained with IV infusions. Serum IgG levels above $5 \mathrm{~g} / 1$ are generally accepted as the minimal protective threshold although trough levels of at least $7 \mathrm{~g} / \mathrm{l}$ may be required to achieve adequate protection against infections for some patients [25-27]. In the present study, trough levels were substantially higher than this 
Table 5 Infusions associated with tolerability concerns or AEs

\begin{tabular}{lllll}
\hline & \multicolumn{2}{l}{ IGSC 20\% treatment dose } & \\
\cline { 2 - 5 } & $145 \%$ of IGIV & Adjusted & Individualized & Overall \\
\hline Total infusions $(n)$ & 731 & 867 & 2729 & 4327 \\
Rate reduced, $n(\%)$ & $1(0.1)$ & $4(0.5)$ & $0(0.0)$ & $5(0.1)$ \\
Interrupted, $n(\%)$ & $0(0.0)$ & $1(0.1)$ & $1(0.0)$ & $2(0.0)$ \\
Stopped, $n(\%)$ & $0(0.0)$ & $0(0.0)$ & $1(0.0)$ & $1(0.0)$ \\
No reduction, interruption or stop, $n(\%)$ & $730(99.9)$ & $862(99.4)$ & $2727(99.9)$ & $4319(99.8)$ \\
\hline
\end{tabular}

accepted protective threshold with a median serum IgG trough level above $14 \mathrm{~g} / 1$ throughout IGSC $20 \%$ treatment. This may have been due in part to the relatively high $\mathrm{Ig}$ doses that patients had been receiving prior to study onset. Indeed, as per study design, the patients received the same monthly $\mathrm{IgG}$ dose/ $\mathrm{kg}$ in period 1 as they did prior to study entry. Therefore, the dose of IGIV $10 \%$ was not predefined per protocol but was determined by the treating physician and the IGSC $20 \%$ dose was subsequently adjusted to achieve systemic exposure equivalent to the IGIV $10 \%$ treatment dose. Although high, the mean weekly dose of IGSC $20 \%$ dose in this study was within the range $(0.177-0.224 \mathrm{~g} / \mathrm{kg} \mathrm{BW}$ per week) reported for another IGSC $20 \%$ product [11].

The high serum IgG trough levels maintained throughout the study were efficacious in preventing VASBIs as demonstrated by the low annualized rate of VASBIs per patient during IGSC $20 \%$ treatment. This rate of VASBIs was significantly lower than the threshold specified by the FDA and EMA guidelines as providing substantial evidence of efficacy $[19,28,29]$. A further indicator of the protective effect of IGSC $20 \%$ was the annualized frequency of any infections (2.41 events/patient), which was comparable to the incidence of infections (2.76 events /patient) reported for another IGSC
$20 \%$ preparation [11] and lower than the annualized rate of any infection episodes reported with other licensed IGSC products: 3.946 events/patient in a 6-month study with IGSC $16 \%$ [30] or 4.1 events/patient with an IGSC $10 \%$ product [12], although differences in study design and product concentrations may limit direct comparison. These efficacy results, together with the positive outcomes obtained for the additional efficacy assessments (number and duration of fever episodes and of hospitalizations as well as the low rate of days missed from work, school, or daily activity), further support the protective effect of IGSC $20 \%$ as replacement therapy in PIDD.

While the SC administration of $\operatorname{IgG}$ has been associated with fewer systemic AEs than IGIV, a higher incidence of local AEs has been reported [31]. The incidence of systemic AEs related to IGSC $20 \%$ infusions in the present study was about 11 -fold lower than for IGIV $10 \%$ treatment. The rate per infusion of local AEs deemed related to IGSC $20 \%(0.015$ event/infusion) was much lower than the rates reported with a licensed equivalent IGSC $20 \%$ preparation in studies conducted in the USA (0.592 and 0.600 event/infusion, respectively) [11, 32] and in Japan (0.274 event/infusion) [33] and lower than the rates observed in an EU study (0.060 event/

Table 6 Pharmacokinetic parameters for the IGSC $20 \%$ and IGIV $10 \%$ treatments

\begin{tabular}{|c|c|c|c|c|c|c|c|c|}
\hline \multirow{2}{*}{$\begin{array}{l}\text { Treatment Period }{ }^{\mathrm{a}} \text { (IP) } \\
\text { Dosing interval }\end{array}$} & \multicolumn{4}{|c|}{ Period 1 (IGIV $10 \%$ ) } & \multirow{2}{*}{\multicolumn{2}{|c|}{$\begin{array}{l}\text { Period } 2 \text { (IGSC } 20 \%, \\
145 \% \text { of IGIV } 10 \%) \\
1 \text { week }(n=18)\end{array}$}} & \multirow{2}{*}{\multicolumn{2}{|c|}{$\begin{array}{l}\text { Period } 4 \text { (IGSC } 20 \%, \\
\text { individualized) } \\
1 \text { week }(n=60)\end{array}$}} \\
\hline & 3 weeks $(n=$ & 16) & 4 weeks $(n=$ & 38) & & & & \\
\hline Parameter [unit] & Geom. mean & $95 \% \mathrm{CI}$ & Geom. mean & $95 \% \mathrm{CI}$ & Geom. mean & $95 \% \mathrm{CI}$ & Geom. mean & $95 \% \mathrm{CI}$ \\
\hline AUC (g/days/l) & 352.05 & $\begin{array}{r}319.74 \\
387.63\end{array}$ & 410.40 & $\begin{array}{r}381.24- \\
441.79\end{array}$ & 108.33 & $97.60-120.24$ & 115.21 & $\begin{array}{r}109.23- \\
121.52\end{array}$ \\
\hline $\begin{array}{c}\text { AUC / (Dose/Weight) } \\
{[(\mathrm{g} / \text { days } / 1) /(\mathrm{g} / \mathrm{kg})]}\end{array}$ & 606.99 & $\begin{array}{r}495.29- \\
743.87\end{array}$ & 796.30 & $\begin{array}{r}715.36- \\
886.40\end{array}$ & 472.50 & $\begin{array}{r}411.07- \\
543.10\end{array}$ & 536.05 & $\begin{array}{r}495.58- \\
579.82\end{array}$ \\
\hline Clearance $^{\mathrm{b}}[\mathrm{ml} / \mathrm{kg} /$ days $]$ & 1.65 & $1.34-2.02$ & 1.26 & $1.13-1.40$ & 2.12 & $1.84-2.43$ & 1.87 & $1.72-2.02$ \\
\hline $\mathrm{Cmax}(\mathrm{g} / \mathrm{l})$ & 27.09 & $24.30-30.19$ & 24.85 & $23.18-26.64$ & 17.31 & $15.11-19.82$ & 19.31 & $18.13-20.57$ \\
\hline $\operatorname{Tmax}(\mathrm{h})$ & 6.94 & $3.96-12.17$ & 5.84 & $3.62-9.40$ & 54.19 & $36.28-80.92$ & 78.68 & $65.37-94.70$ \\
\hline $\mathrm{Cmin}(\mathrm{g} / \mathrm{l})$ & 12.03 & $10.64-13.60$ & 10.37 & $9.50-11.33$ & 13.85 & $12.61-15.21$ & 14.00 & $13.14-14.91$ \\
\hline
\end{tabular}

$95 \%$ CI $95 \%$ confidence interval

${ }^{\text {a }}$ Period 1 and period 2 data includes patients aged 12 years and older; period 4 data includes patients aged 2 years and older.

${ }^{\mathrm{b}}$ Apparent clearance for $\mathrm{SC}$ administration 
Table 7 Trough levels of total IgG at the end of treatment periods

\begin{tabular}{|c|c|c|c|c|c|}
\hline \multicolumn{2}{|c|}{ Treatment interval } & Patient number $(n)$ & Geometric mean $(95 \% \mathrm{CI})$ & Median $(95 \% \mathrm{CI})$ & Min; $\max$ \\
\hline \multicolumn{6}{|l|}{ IGIV $10 \%$} \\
\hline 3 weeks & & 19 & $11.58(10.36-12.94)$ & $12.00(11.00-14.10)$ & $5.45 ; 14.50$ \\
\hline 4 weeks & & 50 & $10.19(9.55-10.88)$ & $10.20(9.61-11.30)$ & $6.09 ; 18.50$ \\
\hline \multicolumn{6}{|c|}{ IGSC $20 \%$} \\
\hline 1 week & $145 \%$ of IV dose & 27 & $14.77(13.86-15.74)$ & $15.30(12.80-16.10)$ & $12.00 ; 20.30$ \\
\hline 1 week & Adjusted dose (145\% of IV dose) & 66 & $14.20(13.48-14.96)$ & $14.65(13.80-15.60)$ & $8.65 ; 22.20$ \\
\hline 1 week & Individualized dose $\mathrm{e}^{\mathrm{a}}$ & 57 & $14.74(14.03-15.48)$ & $15.10(14.00-16.40)$ & $8.81 ; 23.40$ \\
\hline
\end{tabular}

$95 \%$ CI $95 \%$ confidence interval

${ }^{a}$ Determined for each patient by comparing the individual serum IgG trough level attained in period 3 to the expected increase in serum IgG trough level calculated from the PK data from periods 1 and 2

infusion) [34]. Consistent with these data, IGSC $20 \%$ treatment was well tolerated, with mostly mild $(92.5 \%)$ or moderate $(7.5 \%)$, and no severe related local AEs reported. No patients discontinued due to a local adverse reaction, and $98.7 \%$ of IGSC $20 \%$ infusions were not associated with any local related non-serious AE. For $99.8 \%$ of IGSC $20 \%$ infusions, no reduction of infusion rate was required; no infusions had to be interrupted or stopped due to AE or tolerability concerns, indicating an overall short-term tolerability at least equivalent to that observed with a similar licensed IGSC $20 \%$ product [33]. The same model of electromechanical syringedriver pump was used in all patients to exclude potential differences in tolerability and local adverse reactions that could arise from different pump selections. Electromechanical syringe-driver pumps were also used in US and EU studies conducted with a licensed IGSC $20 \%$ product $[11,34]$.

The favorable tolerability profile of IGSC $20 \%$ infusions permitted the administration of higher infusion rates and volumes per site: the median maximum IGSC $20 \%$ infusion rate (60 $\mathrm{ml} / \mathrm{h} / \mathrm{site}$; range $4.4-180)$ was above the maximum infusion rate recommended for another IGSC $20 \%$ preparation (15-25 $\mathrm{ml} / \mathrm{h} / \mathrm{site})$ [35]. This infusion rate was achieved in more than half of completed IGSC $20 \%$ infusions without increase in the proportion of infusions associated with local adverse reactions, indicating that the maximum recommended infusion rate of $60 \mathrm{ml} / \mathrm{h} /$ site for this study was well-tolerated. Of note, there was no predominance of any body mass index in patients across all age groups; therefore, the possibility that the favorable tolerability of higher rates and volumes was related to the amount of subcutaneous adipose tissue is unlikely. The similarity of IGSC $20 \%$ osmolality (280-292 mOsm/ $\mathrm{kg}$ ) with the physiological plasma osmolality (280$296 \mathrm{mOsm} / \mathrm{kg}$ ) could partly account for the positive tolerability profile of IGSC $20 \%[36,37]$. By comparison, the osmolality of a licensed IGSC $20 \%$ product is higher $(380 \mathrm{mOsm} /$ $\mathrm{kg}$ ) [38]. The use of glycine rather than proline as stabilizer in the investigated IGSC $20 \%$ product may also contribute to its improved tolerability.
The high infusion rates enabled a lower infusion duration (median $0.95 \mathrm{~h}$, range $0.2-6.4 \mathrm{~h}$ ) for IGSC $20 \%$, which is markedly shorter than the duration reported for weekly infusions of a licensed IGSC $20 \%$ preparation $(2 \mathrm{~h}$; range 0.5 $17.0 \mathrm{~h}$ ) [11]. In addition, infusion volumes of up to $60 \mathrm{ml} / \mathrm{site}$ were administered in this study, resulting in one or two sites per IGSC $20 \%$ administration in over $75 \%$ of infusions compared to five or fewer sites in $75 \%$ of infusions with a licensed IGSC $10 \%$ product [12]. The viscosity of IGSC $20 \%$ is similar to those of licensed IGSC 16 and $20 \%$ preparations (14.4 versus $14.4 \mathrm{mPa} / \mathrm{s}$ and $14.7 \mathrm{mPa} / \mathrm{s}$, respectively). In addition, there was no specified needle length for infusions. Regardless, infusion characteristics determined for the study product favored shorter infusion duration and fewer infusion sites. Infusion sites were rotated to avoid any single infusion site being used repeatedly within a short time interval.

Patients across all ages adhered to the SC administration of treatment as evidenced by the overall high rate of study completion; $90 \%$ of patients treated with IGSC $20 \%$ completed the study, including $95 \%$ of patients aged 2 to $<16$ years old, suggesting that IGSC $20 \%$ did not place an unreasonable burden on the daily activities of the adult and pediatric patients. Results of patient-reported outcome measures also showed an overall positive evaluation of IGSC $20 \%$ treatment; in particular, a significant increase in treatment satisfaction in terms of treatment convenience and interference was observed. Home infusion was adopted by a high proportion (96\%) of patients and may have contributed to the perception of enhanced convenience. These results, in line with several reports from other studies, show that the treatment experience of patients with PIDD improves as a result of the practice of subcutaneous administration (reviewed by Wasserman [5]).

In conclusion, IGSC $20 \%$ administered SC at an individually adjusted dose was shown to have an excellent safety and tolerability profile in patients with PIDD. In addition, the low incidence of infections and the maintenance of protective trough levels for total serum IgG demonstrate the efficacy of IGSC $20 \%$ treatment. Excellent tolerability across all age 
groups enabled infusions to be administered at higher rates and volumes compared to conventional SC preparations, leading to shorter infusion durations and fewer infusion sites with a reflection onto patient experience in terms of treatment interference and convenience.

Acknowledgments The study was funded by Baxalta, now part of Shire. The authors thank James N. Moy, Ralph Shapiro, Alan P. Knutsen, Lisa Kobrynski, Richard L. Wasserman, Arye Rubinstein, Elie Haddad, and Douglas H. Jones, investigators in this study, and Tschung-I (Jenny) Ho, Miranda Chapman, Jennifer Doralt, as well as Diane Ito and Lisa Meckley, for their contributions to the review of this manuscript.

Authors' Contributions DG, SF, BM and HL contributed to study conception and design; CB wrote the manuscript; DS, MS, SG, IH, IM, $\mathrm{KP}, \mathrm{AD}, \mathrm{BM}, \mathrm{HL}$, and $\mathrm{LY}$ contributed to study conduction, as well as acquisition and interpretation of data; CB, SF, BM, HL, and LY interpreted the data; SF performed the statistical analysis; and all authors reviewed the manuscript and approved the final version.

Compliance with Ethical Standards This prospective, open-label clinical trial (registered on clinicaltrials.gov: NCT no. 01218438) was conducted in accordance with the Declaration of Helsinki and the international standards of Good Clinical Practice. Informed consent was obtained from each patient prior to undergoing any study procedures.

Conflict of Interest DS, MS, SG, IH, KP, and AD worked as investigators on this Baxalta, now part of Shire-funded clinical study. DS, MS, and KP are on the Baxalta, now part of Shire advisory board and MS and KP have worked as speakers for Baxalta, now part of Shire. SG serves as an ad hoc advisor for Baxalta. CB, BM, HL, DG, and LY are full-time employees at Baxalta, now part of Shire. SF was a full-time employee of Baxalta, now part of Shire, at the time when this study was underway. BM, HL, and LY are shareholders of Baxalta, now part of Shire.

Research involving human participants All procedures performed in the study were in accordance with the ethical standards of the institutional and /or national research committee and with the 1964 Helsinki declaration and its later amendments or comparable ethical standards.

Open Access This article is distributed under the terms of the Creative Commons Attribution 4.0 International License (http:// creativecommons.org/licenses/by/4.0/), which permits unrestricted use, distribution, and reproduction in any medium, provided you give appropriate credit to the original author(s) and the source, provide a link to the Creative Commons license, and indicate if changes were made.

\section{References}

1. Bousfiha A, Jeddane L, Al-Herz W, Ailal F, Casanova JL, Chatila T. The 2015 IUIS phenotypic classification for primary immunodeficiencies. J Clin Immunol. 2015;35:727-38.

2. Bonilla FA, Khan DA, Ballas ZK, Chinen J, Frank MM, Hsu JT. Practice parameter for the diagnosis and management of primary immunodeficiency. J Allergy Clin Immunol. 2015;136:11861205.e78.

3. Modell V, Quinn J, Orange J, Notarangelo LD, Modell F. Primary immunodeficiencies worldwide: an updated overview from the Jeffrey Modell Centers Global Network. Immunol Res. 2016;64: $736-53$.
4. Bruton OC. Agammaglobulinemia. Pediatrics. 1952;9:722-8.

5. Wasserman RL. Progress in gamma globulin therapy for immunodeficiency: from subcutaneous to intravenous infusions and back again. J Clin Immunol. 2012;32:1153-64.

6. American Academy of Allergy, Asthma and Immunology. Eight guiding principles for effective use of IVIG for patients with primary immunodeficiency. 7. 2011. American Academy of Allergy, Asthma and Immunology (AAAAI). Ref Type: Report

7. Melamed I, Testori A, Spirer Z. Subcutaneous immunoglobulins: product characteristics and their role in primary immunodeficiency disease. Int Rev Immunol. 2012;31:451-61.

8. Gardulf A, Hammarström L, Smith CI. Home treatment of hypogammaglobulinaemia with subcutaneous gammag lobulin by rapid infusion. Lancet. 1991;338:162-6.

9. Gardulf A, Andersen V, Björkander J, Ericson D, Frøland SS. Gustafson R and others. Subcutaneous immunoglobulin replacement in patients with primary antibody deficiencies: safety and costs. Lancet. 1995;345:365-9.

10. Gardulf A, Hammarström L. Subcutaneous administration of immunoglobulins. What are the advantages? Clin Immunother. 1996;6:108-16.

11. Hagan JB, Fasano MB, Spector S, Wasserman RL, Melamed I, Rojavin MA. Efficacy and safety of a new 20\% immunoglobulin preparation for subcutaneous administration, IgPro20, in patients with primary immunodeficiency. J Clin Immunol. 2010;30:734 45.

12. Wasserman RL, Melamed I, Kobrynski L, Strausbaugh SD, Stein MR, Sharkhawy M. Efficacy, safety, and pharmacokinetics of a $10 \%$ liquid immune globulin preparation (GAMMAGARD LIQUID, 10\%) administered subcutaneously in subjects with primary immunodeficiency disease. J Clin Immunol. 2011;31:323-31.

13. Al-Herz W, Bousfiha A, Casanova JL, Chapel H, Conley ME, Cunningham-Rundles C. Primary immunodeficiency diseases: an update on the classification from the International Union of Immunological Societies Expert Committee for Primary Immunodeficiency. Front Immunol. 2011;2:54.

14. Conley ME, Notarangelo LD, Etzioni A. Diagnostic criteria for primary immunodeficiencies. Representing PAGID (PanAmerican Group for Immunodeficiency) and ESID (European Society for Immunodeficiencies). Clin Immunol. 1999;93:190-7.

15. Hamamoto Y, Harada S, Kobayashi S, Yamaguchi K, Iijima H, Manabe S. A novel method for removal of human immunodeficiency virus: filtration with porous polymeric membranes. Vox Sang. 1989;56:230-6.

16. Yuasa T, Ishikawa G, Manabe S, Sekiguchi S, Takeuchi K, Miyamura T. The particle size of hepatitis $\mathrm{C}$ virus estimated by filtration through microporous regenerated cellulose fibre. J Gen Virol. 1991;72:2021-4.

17. Hämäläinen E, Suomela H, Ukkomen P. Virus inactivation during intravenous immunoglobulin production. Vox Sang. 1992;63:6-11.

18. Kempf C, Jentsch P, Poirier B, Barre-Sinoussi F, Morgenthaler JJ, Morell A. Virus inactivation during production of intravenous immunoglobulin. Transfusion. 1991;31:423-7.

19. U.S. Department of Health and Human Services, Food and Drug Administration, and Center for Biologics Evaluation and Research. Guidance for industry: safety, efficacy, and pharmacokinetic studies to support marketing of immune globulin intravenous (human) as replacement therapy for primary humoral immunodeficiency. 17. 2008. U.S. Department of Health and Human Services, Food and Drug Administration (FDA). Ref Type: Report

20. Daly PB, Evans JH, Kobayashi RH, Kobayashi AL, Ochs HD, Fischer SH. Home-based immunoglobulin infusion therapy: quality of life and patient health perceptions. Ann Allergy. 1991;67:504 10 .

21. Nicolay U, Haag S, Eichmann F, Herget S, Spruck D, Gardulf A. Measuring treatment satisfaction in patients with primary 
immunodeficiency diseases receiving lifelong immunoglobulin replacement therapy. Qual Life Res. 2005;14:1683-91.

22. Bharmal M, Payne K, Atkinson MJ, Desrosiers MP, Morisky DE, Gemmen E. Validation of an abbreviated treatment satisfaction questionnaire for medication (TSQM-9) among patients on antihypertensive medications. Health Qual Life Outcomes. 2009;7:36.

23. Jolles S, Orange JS, Gardulf A, Stein MR, Shapiro R, Borte M. Current treatment options with immunoglobulin $G$ for the individualization of care in patients with primary immunodeficiency disease. Clin Exp Immunol. 2015;179:146-60.

24. Kobrynski L. Subcutaneous immunoglobulin therapy: a new option for patients with primary immunodeficiency diseases. Biologics. 2012;6:277-87.

25. Lucas M, Lee M, Lortan J, Lopez-Granados E, Misbah S, Chapel $\mathrm{H}$. Infection outcomes in patients with common variable immunodeficiency disorders: relationship to immunoglobulin therapy over 22 years. J Allergy Clin Immunol. 2010;125:1354-60. e1-e4.

26. Orange JS, Grossman WJ, Navickis RJ, Wilkes MM. Impact of trough IgG on pneumonia incidence in primary immunodeficiency: a meta-analysis of clinical studies. Clin Immunol. 2010;137:21-30.

27. Shehata N, Palda V, Bowen T, Haddad E, Issekutz TB, Mazer B. The use of immunoglobulin therapy for patients with primary immune deficiency: an evidence-based practice guideline. Transfus Med Rev. 2010;24(1):S28-50.

28. Committee for Human Medicinal Products. Guideline on the clinical investigation of human normal immunoglobulin for intravenous administration (IVIg) - Draft. EMA/CHMP/BPWP/94033/ 2007 - rev. 2, 14. 2010. London, European Medicines Agency (EMA EMEA). Ref Type: Report

29. Committee for Medicinal Products for Human Use. Guideline on the clinical investigation of human normal immunoglobulin for subcutaneous and/or intramuscular administration (SCIg/IMIg). EMA/CHMP/BPWP/410415/2011 rev 1, 10. 23-7-2015. European Medicines Agency (EMEA) Ref Type: Report
30. Borte M, Quinti I, Soresina A, Fernandez-Cruz E, Ritchie B, Schmidt DS. Efficacy and safety of subcutaneous Vivaglobin ${ }^{\circledR}$ replacement therapy in previously untreated patients with primary immunodeficiency: a prospective, multicenter study. J Clin Immunol. 2011;31:952-61.

31. Berger M. Adverse effects of IgG therapy. J Allergy Clin Immunol Pract. 2013;1:558-66.

32. Niebur HB, Duff CM, Shear GF, Nguyen D, Alberdi TK, Dorsey MJ, et al. Efficacy and tolerability of $16 \%$ subcutaneous immunoglobulin compared with $20 \%$ subcutaneous immunoglobulin in primary antibody deficiency. Clin Exp Immunol. 2015;181:441-50.

33. Kanegane H, Imai K, Yamada M, Takada H, Ariga T, Bexon M. Efficacy and safety of IgPro20, a subcutaneous immunoglobulin, in Japanese patients with primary immunodeficiency diseases. J Clin Immunol. 2014;34:204-11.

34. Jolles S, Bernatowska E, De Gracia J, Borte M, Cristea V, Peter HH. Efficacy and safety of Hizentra ${ }^{\mathbb{R}}$ in patients with primary immunodeficiency after a dose-equivalent switch from intravenous or subcutaneous replacement therapy. Clin Immunol. 2011;141:90-102.

35. CSL Behring. Hizentra, Immune globulin subcutaneous (human), 20\% liquid. 9. 2013.

36. Lemm G. Composition and properties of IVIg preparations that affect tolerability and therapeutic efficacy. Neurology. 2002;59: S28-32.

37. Dychter SS, Ebel D, Mead TR, Yocum RC. Comparison of the tolerability of recombinant human hyaluronidase + normal saline and recombinant human hyaluronidase + lactated ringer's solution administered subcutaneously: a phase IV, double-blind, randomized pilot study in healthy volunteers. Curr Ther Res Clin Exp. 2009;70: 421-38.

38. CSL Behring. Hizentra, immune globulin subcutaneous (human), $20 \%$ liquid. Summary of products characteristics. Link: http:// www.ema.europa.eu/docs/en_GB/document_library/EPAR_Product_Information/human/002127/WC500107057.pdf 